Advances in Dynamical Systems and Applications (ADSA).

ISSN 0973-5321, Volume 15, Number 1, (2020) pp. 101-114

(C) Research India Publications

https://dx.doi.org/10.37622/ADSA/15.1.2020.101-114

\title{
A Factorization of Smooth Maps on Manifolds
}

\author{
Fernando Mejías $^{* 1}$ and Jorge Vielma ${ }^{2}$ \\ 1,2 Professor of Mathematics, ESPOL Polytechnic University, Escuela Superior \\ Politécnica del Litoral, Facultad de Ciencias Naturales y Matemíicas, Campus \\ Gustavo Galindo Km. 30.5 Vía Perimetral, P.O. Box 09-01-5863, Guayaquil, Ecuador.
}

\begin{abstract}
In this paper a generalization of the theorem of Mitchell-Rubel is proved, namely: If $M$ is an open, complete, and simply connected Riemannian manifold with nonpositive sectional curvature then every smooth map from $M$ to $M$ can be factored as an expansion followed by a contraction.
\end{abstract}

Keywords: Smooth maps, Riemannian manifolds, sectional curvature.

2010 Mathematics Subject Classification: 53A03, 58A05.

\section{INTRODUCTION}

In 1988 Mitchell and Rubel published a short article in which they proved several beautiful results in advanced calculus about the possibility of factoring smooth maps, see [3]. Their main theorem, characterized by a nice geometrical flavor, establishes that if $F: \mathbb{R}^{n} \rightarrow \mathbb{R}^{n}$ is a smooth map, then $F$ can be expressed as the composite of a contraction with an expansion, that is, there are two smooth maps $C, E: \mathbb{R}^{n} \rightarrow \mathbb{R}^{n}$ such that

$$
F=C \circ E,
$$

with

$$
|C(x)-C(y)| \leq|x-y| \leq|E(x)-E(y)|
$$

for all $x, y \in \mathbb{R}^{n}$. Furthermore, they exhausted all the possibilities of related propositions in the setting of Euclidean spaces. Additionally, in a remark (pg. 714)

\footnotetext{
${ }^{*}$ Corresponding author.
} 
they established that a generalization of the result can be set in the context of smooth manifolds.

What follows is a proof of the general version of the theorem of Mitchell and Rubel, presented as a motivational illustration problem for the outline of a course in differential geometry which would combine classical and modern topics of the subject, and whose requirements are basic elements of advanced calculus as in Spivak [6], with some basics of topology of metric spaces as in Kaplansky [1] or Munkres [4]. Here we highlight the geometric faces of the main points of the subject that are directly related to the problem.

\section{PRELIMINARIES FROM CALCULUS}

Because the setting of the theorem of Mitchell-Rubel is in the context of Euclidean space and given the plethora of references available, each with its own conventions about notation, and just to have a uniform presentation on this matter, we state some key points about the subject, in any case we adopt the notation, results, etc., from Spivak [6].

For $x \in \mathbb{R}^{n}$ we denote by $|x|$ the norm of $x$ and by $\langle x, y\rangle$ the inner product between $x$ and $y$. A map $f: \mathbb{R}^{n} \rightarrow \mathbb{R}^{n}$ is called a contraction if

$$
|f(x)-f(y)| \leq|x-y| \text { for all } x, y \in \mathbb{R}^{n} ;
$$

similarly, $f$ is said to be an expansion if

$$
|f(x)-f(y)| \geq|x-y| \text { for all } x, y \in \mathbb{R}^{n} .
$$

Given $p \in \mathbb{R}^{n}$, the tangent space of $\mathbb{R}^{n}$ at $p$ is denoted by $\mathbb{R}^{n}$ and the corresponding norm and inner product by $|\cdot|_{p}$ and $\langle\cdot, \cdot\rangle_{p}$, respectively. For a smooth map $f: \mathbb{R}^{n} \rightarrow \mathbb{R}^{m}$ the derivative at $p$ is a linear map $D f(p): \mathbb{R}^{n} \rightarrow \mathbb{R}^{m}$, which induces a linear map $f_{* p}: \mathbb{R}_{p}^{n} \rightarrow \mathbb{R}_{f(p)}^{m}$ defined by

$$
f_{* p}\left(v_{p}\right)=(D f(p)(v))_{f(p)} .
$$

The collection of all those $f_{* p}$ is denoted by $f_{*}$. For a smooth curve $\gamma:[a, b] \rightarrow \mathbb{R}^{n}$ the length of $\gamma$ is denoted by $\ell_{a}^{b}(\gamma)$ or $\ell(\gamma)$. The following lemma is a consequence of problem 4-14 in Spivak [6].

Lemma 1. Let $f, g: \mathbb{R}^{n} \rightarrow \mathbb{R}^{n}$ be two smooth functions such that

$$
\left|f_{* x}\left(v_{x}\right)\right|_{f(x)} \leq\left|g_{* x}\left(v_{x}\right)\right|_{g(x)},
$$

for all $x \in \mathbb{R}^{n}$. If $\gamma:[a, b] \rightarrow \mathbb{R}^{n}$ a smooth curve then

$$
\ell(f \circ \gamma) \leq \ell(g \circ \gamma)
$$


We will construct some functions depending on the following result.

Lemma 2. Let $F: \mathbb{R}^{n} \rightarrow \mathbb{R}^{n}$ be a smooth map, then there exists a smooth function $\lambda:(-1,+\infty) \rightarrow \mathbb{R}$ such that

(i) $\lambda^{\prime}(r) \geq 0$ for all $r \in(-1,+\infty)$,

(ii) $\lambda(r) \geq \max \left\{\left|F_{*}\left(v_{x}\right)\right|:|x|=r, v_{x} \in \mathbb{R}_{x}^{n}\right.$ and $\left.\left|v_{x}\right|=1\right\}$, and

(iii) $\lambda(r) \geq 1$ for all $r \in(-1,+\infty)$.

Note that the existence of the maximum indicated in part (ii) is warrantied by the fact that that $F$ is smooth and, both the sphere $S^{n-1}(r)$ in $\mathbb{R}^{n}$ and the sphere $S^{n-1}(1)$ in $\mathbb{R}_{p}^{n}$ are compact sets.

\section{THE THEOREM OF MITCHELL-RUBEL}

In this section we study in detail the main result of [3] which is meant to be motivation for the general concepts and techniques that follow in our presentation.

Theorem 3 (Theorem of Mitchell-Rubel). If $F: \mathbb{R}^{n} \rightarrow \mathbb{R}^{n}$ is a map of class $C^{1}$, then there exist two smooth maps $s E, C: \mathbb{R}^{n} \rightarrow \mathbb{R}^{n}$, such that $E$ is an expansion, $C$ is a contraction $C$, with

$$
F=C \circ E \text {. }
$$

Proof. Let us define $E: \mathbb{R}^{n} \rightarrow \mathbb{R}^{n}$ by

$$
E(x)=\lambda(|x|) \cdot x
$$

From lemma 2-(iii) we have

$$
E(x) \geq|x| \quad \text { for all } x \in \mathbb{R}^{n} .
$$

For a fixed $x \in \mathbb{R}^{n}$ let $G: \mathbb{R}^{n} \rightarrow \mathbb{R}^{n}$ be the map given by

$$
G(y)=\lambda(|x|) \cdot y \text {. }
$$

If $|y| \geq|x|$, then $\lambda(|y|) \geq \lambda(|x|)$ by lemma 2-(iii), therefore

$$
|E(x)|=|G(x)| \leq|G(y)|,
$$

also

$$
|G(y)| \leq|\lambda(|y|) \cdot y|=|E(y)| .
$$


Then

$$
|E(x)-E(y)|=|\lambda(|x|) x-\lambda(|y|) y| \geq|\lambda(|x|) x-\lambda(|x|) y|
$$

and

$$
|x-y| \leq \lambda(|x|) \cdot|x-y| \leq|E(x)-E(y)|,
$$

and we have that $E$ is an expansion.

Let $v \in \mathbb{R}^{n}$ with $\langle x, v\rangle \geq 0$, then

$$
|x+v|^{2}=|x|^{2}+2\langle x, v\rangle+|v|^{2},
$$

then $|x+v| \geq|x|$. The map $E$ is smooth because so is $\lambda$, therefore there exists a map $\phi: \mathbb{R}^{n} \rightarrow \mathbb{R}^{n}$ such that $\phi(v) \rightarrow 0$ as $v \rightarrow 0$, and

$$
E(x+v)-E(x)=D E(x)(v)+|v| \phi(v) .
$$

Then for all $v$ with $|v|$ small enough we have

$$
\lambda(|x|) \cdot|v| \leq|E(x+v)-E(x)| \leq|D E(x)(v)| .
$$

But $|v|=\left|v_{x}\right|_{x}$ and $\left|E_{* E(x)}\left(v_{x}\right)\right|_{E(x)}=\left|\left[D E(x)\left(v_{x}\right)\right]_{* E(x)}\right|_{E(x)}$. Thus we have

$$
\lambda(|x|) \cdot\left|v_{x}\right|_{x} \leq\left|E_{* E(x)}\left(v_{x}\right)\right|_{E(x)}
$$

or,

$$
\lambda(|x|) \cdot\left|v_{x}\right| \leq\left|E_{*}\left(v_{x}\right)\right| .
$$

If $\langle x, v\rangle<0$ we obtain the same inequality by applying $E_{*}$ (which is linear) to $-v_{x}$.

For a given $x \in \mathbb{R}^{n}$ and any $v \in \mathbb{R}^{n}$, with $|v| \neq 0$ we have by lemma 2-(ii) and (5)

$$
\left|E_{*}\left(v_{x}\right)\right| \geq \lambda(|x|) \cdot\left|v_{x}\right| \geq\left|F_{*}\left(\frac{v_{x}}{\left|v_{x}\right|}\right)\right| \cdot\left|v_{x}\right|,
$$

thus

$$
\left|E_{*}\left(v_{x}\right)\right| \geq\left|F_{*}\left(v_{x}\right)\right|
$$

Let $x, y \in \mathbb{R}^{n}$ be two arbitrary points and let $a=E^{-1}(x), b=E^{-1}(y)$. Let $L(x, y)$ be line segment connecting $x$ and $y$ an $\varphi:[0,1] \rightarrow \mathbb{R}^{n}$ given by $\varphi(t)=t y+(1-t) x$. Then $\gamma=E^{-1} \circ \varphi$ is a parametric curve connecting $a$ and $b$. By inequality (6) we may apply lemma 1 and get

$$
\ell(F \circ \gamma) \leq \ell(E \circ \gamma)
$$

but

$$
|F(a)-F(b)| \leq \ell(F \circ \gamma) \quad \text { and } \quad \ell(E \circ \gamma)=|E(a)-E(b)|
$$


then

$$
|F(a)-F(b)| \leq|E(a)-E(b)|,
$$

but this last inequality is equivalent to

$$
\left|F\left(E^{-1}(x)\right)-F\left(E^{-1}(y)\right)\right| \leq|x-y|
$$

or

$$
\left|F \circ E^{-1}(x)-F \circ E^{-1}(y)\right| \leq|x-y| .
$$

This means that the map $C=F \circ E^{-1}$ is a contraction. By the chain rule and the inverse function theorem we have that $C$ is smooth, see theorems 2.2 and 2.11 in Spivak [6].

\section{SMOOTH MANIFOLDS}

In order to establish a general version of the theorem of Mitchell-Rubel one could think of metric spaces, where the notions of contraction and expansion may be easily established, in fact if $(M, d)$ is a metric space, a function $E: M \rightarrow M$ is an expansion if

$$
d(x, y) \leq d(E(x), E(y)) \quad \text { for all } x, \in M .
$$

Similarly a function $C: M \rightarrow M$ is said to be a contraction if

$$
d(C(x), C(y)) \leq d(x, y) \quad \text { for all } x, \in M
$$

On the other hand we have that the notion of smoothness in this context, in general, does not make sense. Thus we have to work with metric spaces with some more structure, which somehow look like Euclidean space.

A manifold is a metric space $(M, d)$ such that for each $p \in M$ there exists a neighborhood $U$ of $p$ and a homeomorphism $\phi: U \rightarrow \mathbb{R}^{n}$, for some $n$; we say that $M$ is locally like Euclidean space. The number $n$ is constant on each connected component of $M$ and if $M$ is connected we say that $n$ is the dimension of $M$ (sometimes we write $M^{n}$ to indicate this fact.)

The pair $(\phi, U)$ is known as a coordinate system around $p$. Two of such coordinate systems $(\phi, U)$ and $(\psi, V)$ are said to be $C^{\infty}$-related if the maps

$$
\begin{aligned}
& \phi \circ \psi^{-1}: \psi(U \cap V) \rightarrow \phi(U \cap V), \\
& \psi \circ \phi^{-1}: \phi(U \cap V) \rightarrow \psi(U \cap V)
\end{aligned}
$$


are $C^{\infty}$. A family of mutually $C^{\infty}$-related of coordinate systems $\mathcal{A}=\left\{\left(\phi_{i}, U_{i}\right)\right\}$ such that $\left\{U_{i}\right\}$ is a cover of $M$ is called an atlas of $M$. If $\mathcal{A}$ is a maximal atlas for $M$ we say that is $(M, \mathcal{A})$ a smooth manifold.

If $(M, \mathcal{A})$ and $(N, \mathcal{B})$ are two smooth manifolds, we say that a function $f: M^{n} \rightarrow N^{m}$ is differentiable (or smooth) if for all $(\phi, U) \in \mathcal{A}$ and $(\psi, V) \in \mathcal{B}$, the function $\psi \circ f \circ \phi^{-1}: \mathbb{R}^{n} \rightarrow \mathbb{R}^{m}$ is $C^{\infty}$. If $f$ is a smooth homeomorphism we say that it is a diffeomorphism.

Given a smooth manifold $M$, to each $p \in M$ we may associate an $n$-dimensional vector space $M_{p}$ which called the tangent space at $p$, elements of $M_{p}$ are some special linear operators denoted $v_{p}$. Given a coordinate system $(\phi, U)$ around $p \in M$, a basis for $M_{p}$ is formed by the linear operators

$$
\left.\frac{\partial}{\partial \phi^{1}}\right|_{p},\left.\frac{\partial}{\partial \phi^{2}}\right|_{p}, \ldots,\left.\frac{\partial}{\partial \phi^{n}}\right|_{p} .
$$

Any smooth function $f: M \rightarrow N$ induces a linear map $f_{* p}: M_{p} \rightarrow N_{f(p)}$ for each $p \in M$; the collection of all such maps is denoted by $f_{*}$. Details of all these structures can be found in Spivak [5, chapter I.3].

A vector field on $M$ is function $X$ that assigns to each $p \in M$ an element $X(p) \in M_{p}$. If $(\phi, U)$ is a coordinate system $(\phi, U)$ then there are functions $X^{1}, \ldots, X^{n}: M \rightarrow \mathbb{R}$ such that

$$
X(p)=\left.\sum_{i=1}^{n} X^{i}(p) \frac{\partial}{\partial \phi^{i}}\right|_{p} .
$$

The vector field is said to be continuous (smooth) if all the $X^{i}$ are continuous (smooth). The collection of all the smooth vector fields is denoted by $\mathfrak{X}(M)$.

A parametrized curve in a smooth manifold $M$ is a smooth function $\gamma: I \rightarrow M$, for some interval $I$. A vector field $V$ along a curve $\gamma$ is a map such that $V(t) \in M_{\gamma(t)}$ for each $t \in I$, in particular, the velocity of $\gamma$ is the vector field $\frac{d \gamma}{d t}$ along $\gamma$ given by

$$
\frac{d \gamma}{d t}=\gamma_{*} \frac{d}{d t}
$$

\section{CURVATURE}

As we said before, a smooth $n$-manifold $M$ is a metric space which is locally diffeomorphic to $\mathbb{R}^{n}$, now we want to get a sort of a measure of the difference between the two spaces, to do so we start from the geometric notion that Euclidean spaces are 
flat, therefore we want to know how far is $M$ of being as flat as $\mathbb{R}^{n}$, from this idea we get the notion of "curvature."

For a smooth curve in $\gamma:[0, L] \rightarrow \mathbb{R}^{2}$ parametrized by arc-length (which is a smooth 1-manifold) the unit tangent vector at $s$ is $\mathbf{t}(s)=\gamma^{\prime}(s)$ and the normal $\mathbf{n}(s)$ the unit vector which is orthogonal to $\mathbf{t}(s)$ and $[\mathbf{t}(s), \mathbf{n}(s)]$ is the standard orientation. The curvature of $\kappa(s)$ of $\gamma$ at $s$ is defined by

$$
\mathbf{t}^{\prime}(s)=\kappa(s) \cdot \mathbf{n}(s)
$$

If $M$ is a surface in $\mathbb{R}^{3}$, for $p \in M$ we can find a coordinate system $(\phi, \mathcal{O})$ around $p$ such that $x^{3}=f\left(x^{1}, x^{2}\right)$ for a map $f: \phi(\mathcal{O}) \rightarrow \mathbb{R}$ and $\left(x^{1}, x^{2}\right) \in \mathcal{O}$. Let $L$ be a straight line through $p$ parallel to the $x^{3}$-axis. Then if $\Pi$ is a plane containing $L, \Pi \cap M$ is a plane curve. A theorem by Euler establishes that among all the possible planes $\Pi$ there are two $\Pi_{1}$ and $\Pi_{2}$ such that the curvatures at $p$ of the corresponding curves $\Pi_{1} \cap M$ and $\Pi_{2} \cap M$ have the maximum and the minimum values, see Spivak [5, theorem II.2.1]. Those numbers are called the principal curvatures of $M$ at $p$.

In 1827 Gauss published a paper in Latin which is probably the most important single research in differential geometry: Disquisitions generales circa superficies curvas, where he introduced the concept of curvature. It can be proved that for any $p \in M^{2}$, the Gaussian curvature $K(p)$ is given by

$$
K(p)=k_{1}(p) \cdot k_{2}(p) .
$$

For example, let us consider the following smooth 2-manifolds. First, the Euclidean space $\mathbb{R}^{2}$ we have that $k_{1}=k_{2}=0$, therefore $K \equiv 0$. For the sphere $S^{2}(a)$ (radius $a$ ), $k_{1}=k_{2}=1 / a$ (the curvature of great circles on $S^{2}(a)$ ), thus $K \equiv 1 / a^{2}$. A 2-manifold with nonpositive curvature is obtained by considering the Poincaré plane:

$$
\mathbb{R}_{+}^{2}=\left\{\left(x^{1}, x^{2}\right): x^{2}>0\right\}
$$

in which "lines" are the arcs of circles with center on the $x^{1}$-axis and the segments of the form $\left\{\left(a, x^{2}\right): x^{2}>0\right\}$.

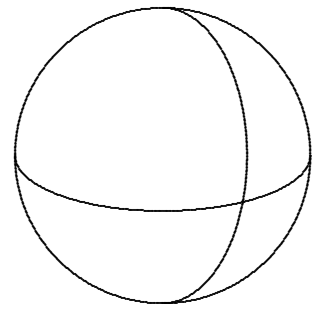

The sphere $S^{2}$

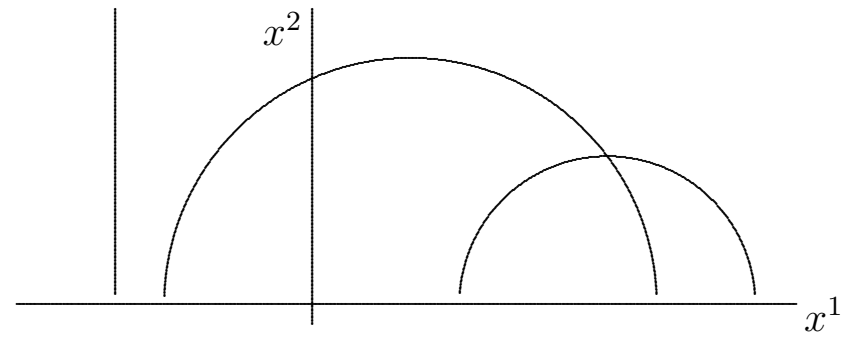

The Poincaré plane 


\section{RIEMANNIAN METRICS}

The notion of a manifold was introduced in 1854 by Bernhard Riemann in his paper Über die Hypothesen, welche der Geometric zu Grunde liegen (On the Hypotheses which lie at the Foundations of Geometry), in order to establish a general version of curvature. For translation into English of Riemann's work as well as the cited Gauss' paper with an exhaustive analysis of both of them see Spivak [5, chapters II.3 and II.4]. Let $M$ be smooth manifold. A Riemannian metric is function $\langle$,$\rangle that assigns to each$ $p \in M$ a positive definite inner product $\langle,\rangle_{p}$ on $M_{p}$, and it induces a norm $\|\cdot\|_{p}$ on $M_{p}$ for each $p \in M$.

The following result is a generalization of lemma 1 will be useful in our project.

Lemma 4. Let $f, g: M \rightarrow M$ be two smooth functions on a smooth manifold $M$, such that

$$
\left\|f_{* x}\left(v_{x}\right)\right\|_{f(x)} \leq\left\|g_{* x}\left(v_{x}\right)\right\|_{g(x)},
$$

for all $x \in M$. If $\gamma:[a, b] \rightarrow M$ a smooth curve then

$$
\ell(f \circ \gamma) \leq \ell(g \circ \gamma)
$$

Some more interesting structures can be obtained in this context. Given any two points $p$ and $q$ in a connected Riemannian manifold $M$ it can be proved that there exists a piecewise smooth curve $\gamma:[0,1] \rightarrow M$, therefore we may define

$$
\rho(p, q)=\inf \{\ell(\gamma): \gamma \text { is a piecewise smooth path from } p \text { to } q\} .
$$

It turns out that $\rho$ induces an important structure on $M$ as it is indicated by the following result, see Spivak [5, theorem I.9.7].

Theorem 5. The function $\rho$ is a metric on $M$, and if $d$ is the metric which makes $M a$ manifold, then the spaces $(M, \rho)$ and $(M, d)$ are homeomorphic.

Riemannian metrics give us the opportunity of considering "geodesics" on manifolds, somehow the shortest paths between pairs of points of $M$. For some manifolds a geodesic may be extended from an interval $[a, b]$ to $\mathbb{R}$, in that case we say that $M$ is geodesically complete. After some hard work the following classical result is obtained, see Spivak [5, theorem I.9.18].

Theorem 6 (Theorem of Hopf-Rinow-de Rham). Let $M$ be a Riemannian manifold. Then $M$ is geodesically complete if and only if the space $(M, \rho)$ is complete. Moreover, is $M$ is geodesically complete, for any $p, q \in M$ there is a geodesic of minimal minimal length which connects them. 
In order to proof theorem 6 it is used an existing diffeomorphism $\exp _{p}: \mathcal{O} \rightarrow M$, where $\mathcal{O} \subset M_{p}$ is open, $p \in M$. This "local" diffeomorphism is called the exponential. Similarly, the next result plays will be used as lemma 2 in the proof of theorem 3 .

Lemma 7. Let $M$ be a smooth n-manifold and $F: M \rightarrow M$ be a smooth map and $p \in M$, then there exists a smooth function $\lambda:(-1,+\infty) \rightarrow \mathbb{R}$ such that

(i) $\lambda^{\prime}(r) \geq 0$ for all $r \in(-1,+\infty)$,

(ii) $\lambda(r) \geq \max \left\{\left\|F_{*}\left(v_{x}\right)\right\|: \rho(p, x)=r, v_{x} \in M_{x}\right.$ and $\left.\left\|v_{x}\right\|=1\right\}$, and

(iii) $\lambda(r) \geq 1$ for all $r \in(-1,+\infty)$.

\section{SECTIONAL CURVATURE}

Now we must introduce three new elements which are essential to investigate the geometry of a Riemannian manifold. First, the Lie bracket $[]:, \mathfrak{X}(M) \times \mathfrak{X}(M) \rightarrow$ $\mathfrak{X}(M)$ is defined by

$$
[X, Y](f)=X(Y(f))-Y(X(f))
$$

for all smooth function $f: M \rightarrow \mathbb{R}$.

The second ingredient is the notion of connection which is another operation $\nabla$ : $\mathfrak{X}(M) \times \mathfrak{X}(M) \rightarrow \mathfrak{X}(M)$ which is linear with respect to the vector fields, and whose image at $(X, Y)$, denoted by $\nabla_{X} Y$, is linear over smooth functions on $M$.

Finally the Riemann curvature tensor $R: \mathfrak{X}(M) \times \mathfrak{X}(M) \times \mathfrak{X}(M) \rightarrow \mathfrak{X}(M)$ whose value for $X, Y, Z \in M_{p}$ is denoted by $R(X, Y) Z \in M_{p}$, es defined by

$$
R(X, Y) Z=\nabla_{Y} \nabla_{X} Z-\nabla_{X} \nabla_{Y} Z+\nabla_{[X, Y]} Z
$$

It turns out that for a Riemannian manifold $(M,\langle\rangle$,$) , given p \in M$ and $X, Y \in M_{p}$ are orthonormal, then

$$
\langle R(X, Y) Y, X\rangle=K(p)
$$

where $K$ is the Gaussian curvature of the $\operatorname{surface}^{\exp _{p}}(\mathcal{O})$, and $\mathcal{O}$ is a neighborhood of $0 \in M_{p}$ where $\exp _{p}$ is a diffeomorphism.

The quantity on the left hand side of equation (10) is called the sectional curvature of $M$. The next result by Cartan provides some geometrical information about $M$ assuming a condition on the sign of the sectional curvature and some additional topological hypothesis for $M$. One short proof of Cartan's theorem uses techniques from "Morse theory", a specialized field in differential geometry, and it is proved 
that for each $p \in M$ we have that $\exp _{p}$ is a "global" diffeomorphism, for details see Milnor [2, pg. 102].

Theorem 8 (Theorem of Cartan). Suppose that $M$ is a simply connected, complete Riemannian manifold, and that the sectional curvature is everywhere nonpositive. Then any two points of $M$ are joined by a unique geodesic. Furthermore, $M$ is diffeomorphic to the Euclidean space $\mathbb{R}^{n}$.

The assumption about the sign of the sectional curvature also implies a key point for our project. Let $\gamma, \omega: I \rightarrow M$ be two smooth curves, where $I$ is an interval. Suppose that $p=\gamma\left(t_{0}\right)=\omega\left(t_{0}\right)$ for a $t_{0} \in I$,

$$
\left\langle\gamma_{* p}\left(t_{0}\right), \omega_{* p}\left(t_{0}\right)\right\rangle_{p} \geq 0
$$

For $t_{0}<t_{1}<t_{2}$, let $\beta_{i}$ be the geodesics connecting $\gamma\left(t_{i}\right)$ and $\omega\left(t_{i}\right)$. Then

$$
\ell\left(\beta_{1}\right) \leq \ell\left(\beta_{2}\right)
$$

Therefore if $\rho$ is the metric induced by the Riemannian metric we have

$$
\rho\left(\gamma\left(t_{1}\right), \omega\left(t_{1}\right)\right) \leq \rho\left(\gamma\left(t_{2}\right), \omega\left(t_{2}\right)\right)
$$

\section{THE GENERALIZED THEOREM OF MITCHELL-RUBEL}

We try to develop the prove a generalized version of the theorem of Mitchell-Rubel following the ideas of the original result. Thus, given a smooth map of a manifold $M$ into itself, we will fix $p \in M$ and we need to construct a map which expands $F(x)$ along the geodesic passing through $p$ and $x$. But such a construction may not be possible if, for example, $M$ is compact. Furthermore a local expansion may not extend to a global expansion as happens on the sphere $S^{2}$. Therefore we will work in the context of manifolds as the ones described in Cartan's theorem.

Theorem 9 (Theorem of Mitchell-Rubel on manifolds). For $n \geq 2$, let $M$ be an n-dimensional open, complete, and simply connected Riemannian manifold with nonpositive sectional curvature. If $F: M \rightarrow M$ is a smooth map, then $F$ is a composition of a smooth expansion $E$ followed by a smooth contraction $C$, that is, $F=C \circ E$.

Proof. Let $p \in M$ be an arbitrary point, then by Cartan's theorem $\exp _{p}: M_{p} \rightarrow M$ is a diffeomorphism. Furthermore for any $x \in M$ there exists a unique geodesic $\alpha_{x}$ which connects $p$ with $x$. Let us denote by $\ell_{x}$ the length of the geodesic segment from $p$ to $x$, 
thus we have that $\ell_{x}=\rho(p, x)$ where $\rho$ is the metric induced on $M$ by the Riemannian metric.

Define the map $\operatorname{Exp}_{p}: M \rightarrow M_{p}$ by

$$
\operatorname{Exp}_{p}(x)= \begin{cases}p & \text { if } x=p \\ \ell_{x} \frac{\exp _{p}^{-1}(x)}{\left\|\exp _{p}^{-1}(x)\right\|_{p}} & \text { if } x \neq p .\end{cases}
$$

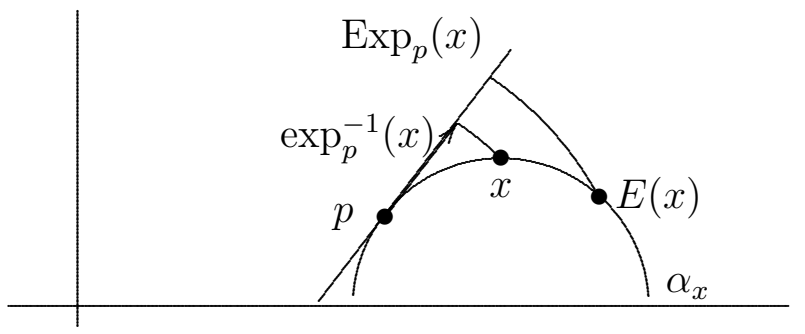

Essentially $\operatorname{Exp}_{p}(x)$ is the unique point in $M_{x}$ which is in the direction of the geodesic $\alpha_{x}$ and $\left|\operatorname{Exp}_{p}(x)\right|=\ell_{x}$.

Now let $\lambda$ be a smooth real function as in lemma 7 and define $E: M \rightarrow M$ by

$$
E(x)=\operatorname{Exp}_{p}^{-1}\left(\lambda\left(\ell_{x}\right) \operatorname{Exp}_{p}(x)\right)
$$

Thus $E(x)$ is the unique point in the geodesic $\alpha_{x}$ such that $\rho(p, E(x))=\lambda\left(\ell_{x}\right) \cdot \ell_{x}$. Clearly $E$ is smooth. Then, from lemma 7-(iii) we have

$$
\rho(p, E(x))=\ell_{E(x)} \geq \ell_{x} \quad \text { for all } x \in M \text {. }
$$

For a fixed $x \in M$ let $G: M \rightarrow M$ be the map given by

$$
G(y)=\operatorname{Exp}_{p}^{-1}\left(\lambda\left(\ell_{x}\right) \operatorname{Exp}_{p}(y)\right)
$$

If $\rho(p, y) \geq \rho(p, x)$, then $\lambda\left(\ell_{y}\right) \geq \lambda\left(\ell_{x}\right)$ by lemma 7-(iii), therefore

$$
\ell_{E(x)}=\ell_{G(x)} \leq \ell_{G(y)},
$$

and

$$
\ell_{G(y)} \leq \lambda\left(\ell_{y}\right) \cdot \ell_{y}=\ell_{E(y)}
$$

Then

$$
\rho(E(x), E(y))=\rho\left(\lambda\left(\ell_{x}\right) \ell_{x}, \lambda\left(\ell_{y}\right) \ell_{y}\right) \geq \rho\left(\lambda\left(\ell_{x}\right) \ell_{x}, \lambda\left(\ell_{x}\right) \ell_{y}\right)
$$


and

$$
\rho(x, y) \leq \lambda(|x|) \cdot r(x, y) \leq \rho(E(x), E(y)),
$$

so we have that $E$ is an expansion.

Let $v_{x} \in M_{x}$ with $\left\langle\alpha_{x}^{\prime}, v_{x}\right\rangle_{p} \geq 0$, where $\langle,\rangle_{p}$ denotes the inner product in $M_{p}$ which is given by the Riemannian metric on $M$.

Therefore, for all $v_{x} \in M_{x}$ with $\left\|v_{x}\right\|$ small enough we have

$$
\lambda\left(\ell_{x}\right) \cdot\left\|v_{x}\right\| \leq\left\|E_{*}\left(v_{x}\right)\right\|
$$

For the case $\left\langle\alpha_{x}^{\prime} v_{x}\right\rangle<0$ is treated using the fact that $E_{*}$ is a linear map.

For $x \in M$ and any $v_{x} \in M$, with $|v| \neq 0$ we have by lemma 7-(ii) and (14)

$$
\left\|E_{*}\left(v_{x}\right)\right\| \geq \lambda\left(\ell_{x}\right) \cdot\left\|v_{x}\right\| \geq\left\|F_{*}\left(\frac{v_{x}}{\left\|v_{x}\right\|}\right)\right\| \cdot\left\|v_{x}\right\|
$$

thus

$$
\left\|E_{*}\left(v_{x}\right)\right\| \geq\left\|F_{*}\left(v_{x}\right)\right\|
$$

For any two points $x, y \in M$ let $a=E^{-1}(x)$ and $b=E^{-1}(y)$. Let $\varphi$ be the geodesic segment connecting $x$ and $y$. Then $\gamma=E^{-1} \circ \varphi$ is a curve connecting $a$ and $b$.

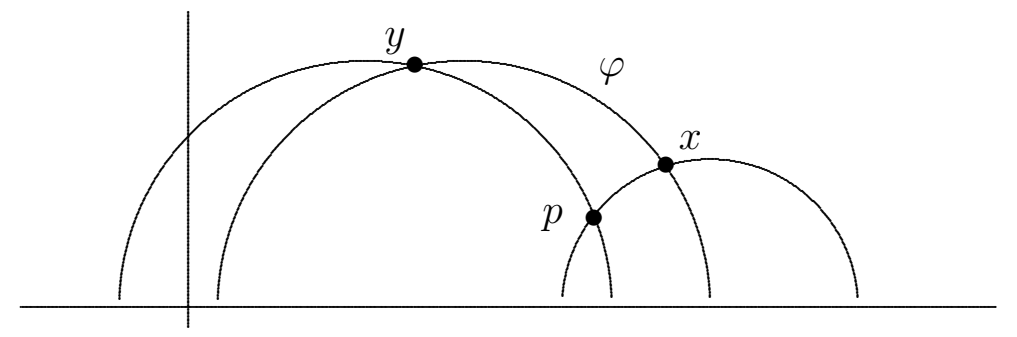

By inequality (15) we may apply lemma 4 and to get

$$
\ell(F \circ \gamma) \leq \ell(E \circ \gamma)
$$

but

$$
\rho(F(a), F(b)) \leq \ell(F \circ \gamma) \quad \text { and } \quad \ell(E \circ \gamma)=\rho(E(a), E(b))
$$

then

$$
\rho(F(a), F(b)) \leq \rho(E(a), E(b)),
$$

but this last inequality is equivalent to

$$
\left|F\left(E^{-1}(x)\right)-F\left(E^{-1}(y)\right)\right| \leq|x-y|,
$$


or

$$
\rho\left(F \circ E^{-1}(x), F \circ E^{-1}(y)\right) \leq \rho(x, y) .
$$

This means that the map $C=F \circ E^{-1}$ is a contraction. By the chain rule and de inverse function theorem we have that $C$ is smooth.

\section{ABOUT THE AUTHORS}

Fernando Mejías received a B.A. degree in Education (major mathematics) from Universidad de Los Andes, Venezuela, in 1985, then he earned a M.Sc. and a Ph.D. degrees (both in mathematics, in 1997 and 2001, respectively) at Northeastern University, Boston, Massachusetts.

He was a member of the faculty of University of the Andes until May 2019, then he became a member of the faculty of the ESPOL Polytechnic University, Ecuador.

Jorge Vielma earned a B.Sc. degree at Utah State University in 1971 and a M.Sc. from Universidad de Oriente (Venezuela, 1978). In 1993 he received a Ph.D. degree in mathematics at the University of Iowa in 1986 and held there a postdoctoral position in 1993.

He worked as a Professor of Mathematics at Universidad de Los Andes, Venezuela, for about 35 years. Currently he is working as a Professor of Mathematics at ESPOL Polytechnic University, Ecuador.

\section{DECLARATION BY THE AUTHORS}

This manuscript has been neither published nor submitted for publication, in whole or in part, either in a serial, professional journal or as a part in a book which is formally published and made available to the public.

\section{REFERENCES}

[1] Kaplansky I., Set theory and metric spaces, (2nd ed.), AMS-Chelsea, Providence, RI, 2001.

[2] Milnor J., Morse theory, Princeton University Press, Princeton, N.J., 1963.

[3] Mitchell J. and Rubel L., Every smooth map of Euclidean space into itself is an expansion followed by a contraction, Amer. Math. Monthly, 95 (1988), 713-716.

[4] Munkres J., Topology, (2nd ed.), Prentice Hall, Upper Saddle River, NJ, 2000. 
[5] Spivak M., A comprehensive introduction to differential geometry, 5volumes, 3rd ed., Publish of Perish, Houston, TX, 2000.

[6]___ Calculus on manifolds, Benjamin, New York, NY, 1965. 